\title{
ELASTICITY AND CRACK PROBLEMS
}

\author{
S. Burhanettin ALTAN
}

I.T.U. School of Civil Engineering Maslak, Istanbul 80626, TURKEY

\section{ABSTRACT}

Definition of a crack in the mathematical theory of elasticity is discussed. Formulation of mode III crack is obtained by Fourier transform technique within the framework of classical elasticity, nonlocal elasticity and gradient-dependent elasticity. A brief discussion is given on the structure of the crack tip in the light of the results obtained.

\section{INTRODUCTI ON}

It is a well-known fact that the fracture mechanics is based on the classical elasticity solution of crack problems which fails to predict the stress and strain field in a core region around a sharp crack tip. Since the singularity in both stress and strain at the crack tip prohibits the use of a fracture criterion based on stresses or strains various ersatz such as stress intensity factor, J-integral, fracture toughness etc. [1] have been invented to circumvent this difficulty. Though these approaches are used successfully for engi neering purposes it is a well-accepted fact that they are far from predicting the state of stress and strain around a crack tip and they do not have a theoretical basis.

One of the main streams of the advancement in science is to extend the fundamental hypotheses of a theory when it proves insufficient in explaining the phenomena in its field. Similar to the other branches of science, in continuum mechanics much effort has been spent in this direction, resulting in new theories. Among them, nonlocal and gradient-dependent theories of elasticty are recently developed ones.

The nonlocal constitutive equations differ from the classical ones in taking into account the nonlocal effects, as its name suggests. In other words the far-reaching character 
of the inter-atomic forces are reflected in the constitutive equations or in continuum mechanics terminology a change in an independent variable at a point causes a change in the dependent constitutive variables not only at the relevant point but also at the points in a neighborhood of it. For detailed information on nonlocal elasticity Kröner [2], Kunin [3], Rogula [4] and Eringen and Edelen [5] can be consulted.

In the gradient-dependent constitutive equations of el astic bodies higher gradients of the deformation field are taken into account $[6,7]$. Though the gradient-dependent elasticity has not been widely used, successful results have been obtained in plasticity by introducing higher gradients of deformation field in the yield function $[8,9]$ resulting finite thickness for stationary shear bands.

The aim of this study is to compare the solutions of mode III crack in classical, nonlocal and gradient-dependent elasticities. In the subsequent section the field equations of linear elasticity are summarized. In the third section a critique on the definition of crack problems in the mathematical theory of elasticity is given and the mode III crack is formulated in terms of crack opening by using Fourier transform technique and the solutions of this problem within the framework of classical elasticity, nonlocal elasticity and gradient-dependent elasticity are given. The final section is devoted to a discussion on the crack tip structure and some further research directions are indicated.

\section{FIELD EQUATIONS OF LINEAR ELASTICITY}

In this section the field equations of linear are summarized and the constitutive equations for nonlocal and gradient-dependent elasticities are field equations of linear, isotropic, homogeneous elasticity consist of the displacement-strain relations

$$
\varepsilon_{i j}(x)=\frac{1}{2}\left[u_{i, j}(x)+u_{j, i}(x)\right] \quad \text { for } \forall x \in B
$$

and the bal ance equations

$$
t_{i j, j}(x)+f_{i}(x)=0 \text { for } \forall x \in B
$$

where $u_{i}, \varepsilon_{i j}, t_{i j}$ denote the Cartesian components of the infinitesimal displacement vector, strain and stress tensors respectively, $\hat{f}_{\imath}$ are the Cartesian components of the body forces and $B$ stands for a bounded, open domain in three dimensional Euclidean space whose closure has a sufficiently smooth boundary and $x$ is the position vector for the material points of the elastic body occupying the region B. Throughout this paper the indicial notation and the summation convention is used i.e. the subscripts will take the value $1,2,3$ and the repeated indices imply summation over the range of indices and moreover 
the indices following a comma indicates the partial derivative with respect to the corresponding position coordinate. The last group of the field equations is the constitutive equations. In this study three different types of constitutive equations will be employed: The first one which will be specified as classical is the Hooke's law

$$
t_{i j}^{c}(x)=\lambda s_{k: k}(x) O_{i j}+2 \mu \varepsilon_{i j}(x) \quad \text { for } \forall x \in B
$$

where $\delta_{i j}$ is the Kronecker delta and $\lambda, \mu$ are Lame constants.

The second type is the nonlocal constitutive equations

$$
t_{i j}^{n}(x)=\int_{B} k\left(\left|x-x^{\prime}\right|, x\right) \sigma_{i j}\left(x^{\prime}\right) d v(x) \text { for } \forall x \in B
$$

where $k$ is the nonlocality kernel which is chosen as $(2 \pi x)^{-4} k_{0}\left(\sqrt{\left(x-x^{\prime}\right) \cdot\left(x-x^{2}\right)} / x\right)$ where $k_{0}$ is the modified Bessel's function of zeroeth order and $x \geq 0$ is a material constant. The third type constitutive equation is the gradient-dependent constitutive equations

$$
t_{i j}^{g}(x)=\sigma_{i j}(x)-\gamma^{2} \sigma_{i j, m m}(x) \quad \text { for } \forall x \in B
$$

where $\sigma_{i j}(x)$ will be found from the classical constitutive relations defined by (3) and $\gamma \geq 0$ is another material constant. Clearly, the nonlocal kernel and the extra material constans $\gamma, x$ are subject to be prescribed by experiments.

\section{MODE III CRACK IN ELASTICITY}

In this section the definition of cracks as a boundary value problem in the linear theory of mathematical elasticity is criticized. Since this critique is specified on mode III crack problem, first, the formulation of this problem in terms of crack opening, will be given.

Consider an infinite elastic medium weakened by a line crack located at $c \leq x \leq c, y=0,-\infty \leq z \leq \infty$ and loaded by a constant traction $\tau$ on the crack surface along the $z$ direction The corresponding boundary value problem is commonly known as antiplane shear problem [10] and is solved by posing a displacement field as follows: $u_{z}=w(x, y)=-w(x,-y)=w(-x, y), u_{x}=0$, $u_{y}=0$. In this case the only nonvanishing strain components are

$$
\varepsilon_{z x}=\frac{1}{2} \frac{\partial w}{\partial x}, \varepsilon_{z y}=\frac{1}{2} \frac{\partial w}{\partial y}
$$


The corresponding stress components are

$$
t_{z x}^{c}=\mu \frac{\partial w}{\partial x} \quad t_{z y}^{c}=\mu \frac{\partial w}{\partial y}
$$

in classical elasticity,

$t_{z x^{\prime}}^{n}(x, y)=\mu(2 \pi x)^{-1} \int_{-\infty}^{\infty} \int_{0} K_{0}\left[\sqrt{\left(x-x^{\prime}\right)^{2}+\left(y-y^{\prime}\right)^{2}} / x\right] \frac{\hat{w}\left(x^{\prime}, y^{\prime}\right)}{\partial x^{\prime}} d x^{\prime} d y^{\prime}$

$t_{z y^{n}}^{n}(x, y)=\mu(2 \pi x)^{-1} \int_{-\infty}^{\infty} \int_{0} k_{0}\left[\sqrt{\left(x-x^{\prime}\right)^{2}+\left(y-y^{\prime}\right)^{2}} / x\right] \frac{\vec{w}\left(x^{\prime}, y^{\prime}\right)}{\partial_{y^{\prime}}} d x^{\prime} d y^{\prime}$

in nonlocal elasticity and

$$
\begin{aligned}
& t_{z x}^{\mathrm{g}}(x, y)=\mu \frac{\partial}{\partial x}\left\{w-\gamma^{2}\left[\frac{\partial^{2} w}{\partial^{2} x}+\frac{\partial^{2} w}{\partial^{2} y}\right]\right\} \\
& t_{z y}^{g}(x, y)=\mu \frac{\partial}{\partial y}\left\{w-\gamma^{2}\left[\frac{\partial^{2} w}{\partial x^{2}}+\frac{\partial^{2} w}{\partial y^{2}}\right]\right\}
\end{aligned}
$$

in gradient-dependent elasticity.

3.1. Formulation of Mode III Crack in Classical Elasticity

Introducing the expressions (7) into the balance equations (2) we obtain

$$
\frac{\partial^{2} w}{\partial x^{2}}+\frac{\partial^{2} w}{\partial y^{2}}=0
$$

By using the Fourier transform technique the solution of this equation and the components of the stress can be written as foll lows:

$$
\begin{aligned}
& w^{c}(x, y)=\sqrt{2 / \pi} \int_{0}^{\infty} A(\xi) \exp (-\xi y) \cos (\xi x) d \xi \\
& t_{z x}^{c}(x, y)=-\mu \sqrt{2 \pi} \int_{0}^{\infty} \xi A(\xi) \exp (-\xi y) \sin (\xi x) d \xi \\
& t_{z y}^{c}(x, y)=-\mu \sqrt{2 / \pi} \int_{i}^{\infty} \xi A(\xi) \exp (-\xi y) \cos (\xi x) d \xi
\end{aligned}
$$

3.2. Formulation of Mode III Crack in Nonlocal Elasticity

For the solution of the same problem in nonlocal elasticity we consider the inverse form of the constitutive equation (4). It can be shown that the constitutive equation is equivalent to the following differential equation:

$$
t_{i j}^{n}(x)-x^{2} t_{i j, m m}^{n}(x)=\sigma_{i j}(x)
$$


In this case the equations ( 8 ) become

$$
\begin{aligned}
& t_{z x}^{n}-x^{2} \nabla^{2} t_{z x}^{n}=\mu \frac{\partial w}{\partial x} \\
& t_{z y}^{n}-x^{2} \nabla^{2} t_{z y}^{n}=\mu \frac{\partial w}{\partial y}
\end{aligned}
$$

where $\nabla$ is the gradient operator. The equations of balance are satisfied if it is assumed that the stress field is derived from a scalar function $\phi(x, y)$ as follows:

$$
t_{z x}=\frac{\partial \phi}{\partial y}, t_{z y}=-\frac{\partial \phi}{\partial x}
$$

Then introducing the strain components obtained from (13) into the incompatibility equation

$$
\frac{\partial \varepsilon_{z x}}{\partial y}-\frac{\partial \varepsilon_{z v}}{\partial x}=D(x, y)
$$

where $D(x, y)$ is the dislocation density and employing (14) we arrive at

$$
\nabla^{2} \phi-\varkappa^{2} \nabla^{4} \phi=\mu D(x, y)
$$

Since we are looking for a solution concerned with a line crack we consider $D(x, y)=-w(x) \delta(y)$ where $\delta$ is the Dirac delta measure. By using the Fourier transform the solution of this equation and the displacement and the stress fields of the problem can be written in the following form:

$\phi=\mu \sqrt{2>\pi} \int_{0}^{\infty} A(\xi)\left\{\exp (-\xi y)-\frac{\xi}{\sqrt{\left(\xi^{2}+x^{-2}\right)}} \exp \left(-\sqrt{\xi^{2}+x^{-2}} y\right)\right\} \cos (\xi x) d \xi$

$w^{n}(x, y)=\sqrt{2 / \pi} \int_{0}^{\infty} A(\xi) \exp (-\xi y) \cos (\xi x) d \xi$

$$
\begin{aligned}
& t_{z x}^{n}(x, y)=-\mu \sqrt{2 / \pi} \int_{0}^{\infty} \xi A(\xi)\left\{\exp (-\xi y)-\exp \left(-\sqrt{\xi^{2}+x^{-2}} y\right\}\right\} \sin (\xi x) d \xi \\
& t_{z y}^{n}(x, y)=-\mu \sqrt{2 / \pi} \int_{0}^{\infty} \xi A(\xi)\left\{\exp (-\xi y)-\frac{\xi}{\left.\sqrt{\left(\xi^{2}+x^{-2}\right)} \exp \left(-\sqrt{\xi^{2}+x^{-2}} y\right)\right\}}\right. \\
& \cos (\xi x) d \xi
\end{aligned}
$$

3. 3. Formulation of Mode III Crack in Gradient Elasticity

$$
\text { Introducing (9) into the equations of balance we abtain }
$$

$$
\nabla^{2} w-\gamma^{2} \nabla^{4} w=0
$$

Again, by using the Fourier transforms the solution of this equation and the stress components can be written as follows: 


$$
\begin{aligned}
& w^{g}(x, y)=\sqrt{2 / \pi} \int_{0}^{\infty} A(\xi)\left\{\frac{\gamma^{-2}}{\xi^{2}+\gamma^{-2}}\right\} \exp (-\xi y) \cos (\xi x) d \xi \\
& t_{z x}^{g}(x, y)=-\mu \sqrt{2}-\pi \int_{0}^{\infty} \xi A(\xi) \exp (-\xi y) \sin (\xi x) d \xi \\
& t_{z y}^{g}(x, y)=-\mu \sqrt{2}-\pi \int_{u}^{\infty} \xi A(\xi) \exp (-\xi y) \cos (\xi x) d \xi
\end{aligned}
$$

In solving the equation (18) the extra boundary condition

$$
\left.\frac{\partial^{2} w}{\partial y^{2}}\right|_{y=0}=0
$$

is used.

All expressions appearing in $(11,17,19)$ are valid for $y \geq 0$.

\section{SOLUTIONS TO MODE III CRACK}

In this section the solutions of made III crack problem corresponding to the three different formulations $(11,17,19)$ will be given. First we wish to discuss the definition of crack problems as a boundary value problem in the mathematical theory of elasticity to prepare a basis for the method which will be employed in this study for solving the problems under consideration. The method that we use in this study will be discussed in full detail elsewhere.

It is a well-known fact that a line crack problem is considered as a mixed boundary vaiue problem in the mathematical theory of elasticity [11]. This definition is based on the fact that the displacement field is continuous on the crack plane outside the crack in classical elasticity. This condition being a consequence of the classical elasticity rather than a requirement which should be imposed for solving crack problems has provided simplicity in the formulation of crack problems. It seems like this condition is treated as a necessary condition for formulating the crack problems in the literature. Now we wish to demonstrate that a crack problem can be solved as a traction boundary value problem. The traction boundary condition which should be satisfied on the crack surface is $\tau_{z y}(x, 0)=\tau$ (const.) $|x| \leq c$ for mode III crack. By (11) $)_{3}$, this condition requires to find a function $A(\xi)$ such that

$$
\mu \sqrt{2} \pi \int_{0}^{\infty} \xi A(\xi) \cos (\xi x) d \xi=\tau \quad|x| \leq c
$$

is satisfied. To find the unknown function $A(\xi)$ we consider the Neumann Series expansion [12]:

$$
\xi A(\xi)=\sum_{n=0}^{\infty} a_{n} J_{n}(c \xi)
$$


where $J_{n}$ are the Bessel's Function of first kind with integer indices and $a_{n}$ are the unknown coefficients. Substituting this expansion in (21) and taking into account the fact that

$$
\int_{0}^{\infty} J_{n}(c \xi) \cos (\xi x) d \xi=\frac{\cos [n \cdot \arcsin (x / c)]}{c \sqrt{1-(x / c)^{2}}} \quad|x| \leq c
$$

$([13], 6.671 .2)$ we arrive at

$$
\sum_{n=0}^{n} a_{n} \frac{\cos [n \arcsin (x / c)]}{c \sqrt{1}-(x / c)^{2}}-\sqrt{\frac{\pi}{2}} \frac{\pi}{\mu}
$$

On the other hand, it can be shown that

$$
\begin{aligned}
& U_{2 k}(x)=(-1)^{k} \frac{\cos [(2 k+1) \arcsin (x)]}{\sqrt{1}-x^{2}} \\
& U_{2 k+1}(x)=\frac{\sin [2(k+1) \arccos (x)]}{\sqrt{1}-x^{2}}
\end{aligned}
$$

Since the right hand side of (24) is a constant we may deduce from (24) that

$$
a_{0}=0, a_{1}=\sqrt{\frac{\pi}{2}} \frac{\tau}{\mu} c, a_{n}=0 \quad n=2,3, \ldots
$$

It is interesting to note that if the right hand side of (24) were symmetric function of $x$ then the unknown function $A(\xi)$ could be expressed in terms of $J_{2 k+1}(c \xi)$ only and since

$$
\int_{0}^{\infty} \xi^{-1} J_{2 n+1}(c \xi) \cos (\xi x) d \xi=0 \quad|x| \geq c
$$

the continuity of the displacement outside the crack is satisfied requiring no extra condition.

\subsection{Solution of Mode III Crack in Nonlocal Elasticity}

To find the solution of the problem under considerartion in nonlocal elasticity we replace $\xi A C \xi)$ by the Neumann Series expansion given by (2Z) in (17), and apply the boundary condition

$$
-\mu \sqrt{2} \pi_{0} \int_{n \neq 0}^{\infty} \sum_{n} J_{n}(c \xi)\left\{1-\frac{\xi}{v\left(\xi^{2}+x^{-2}\right)}\right\} \cos (\xi x) d \xi=-\tau
$$

By multiplying this equation by $\cos [m \cdot \arcsin (x, c)]$ and integrating on the crack surface we obtain

$$
\sum_{n=0}^{\infty} A_{m n}{ }^{a}=\sqrt{\frac{E}{\pi}} \frac{\tau}{\mu}<\left\{\frac{\sin \frac{(m+1) \pi}{\bar{c}}}{m+1}+\frac{\sin \frac{(m-1) \pi}{2}}{m-1} ; m=0,1, \ldots\right. \text { (29) }
$$

where

$$
A_{m n}=\int_{-c}^{c} \int_{0}^{\infty}\left\{1-\frac{\varepsilon}{\sqrt{\left(c \xi^{2}+x^{-2}\right)}}\right\} J_{n}(c \xi) \cos [m \cdot \arcsin (x / c)] \cos (\xi x) d \xi d x
$$


It can be shown that the integrals $A_{m n}$ are uniformly convergent for $x>0$. The theoretical difficulties are apparent in solving the equations given by (29). On the other hand, for an approximate solution one can consider the truncated form of it which can be solved easily. A computer work carried out on this matter indicates that while the boundary condition on crack surface is satisfied better with the contribution of Bessel's functions with even indices crack opening continues outside the crack, in other words there appears discontinuity in the displacement field outside the crack. A full treatment of this problem will be reported elsewhere.

\subsection{Solution of Mode III Crack in Gradient Elasticity}

Since the expressions for the stress components are the same in classical and gradient-dependent elasticities the solution obtained for the classical formulation is valid for the gradient-dependent formulation also. On the other hand the expression for the displacement on the crack plane takes the following form:

$$
w^{g}\left(x, 0^{+}\right)=\frac{\tau}{\mu} \subset \int_{0}^{\infty} \xi^{-1} J_{1}(c \xi)\left\{\frac{\gamma^{-2}}{\xi^{2}+\gamma^{-2}}\right\} \cos (\xi x) d \xi
$$

Since

$$
\int_{0}^{\infty} \xi^{-1} J_{1}(c \xi)\left\{\frac{\gamma^{-2}}{\xi^{2}+\gamma^{-2}}\right\} \cos (\xi x) d \xi=\frac{\pi}{2} I_{1}(c / \gamma) e^{-x / y} \quad x \geq c \quad(31)
$$

where $I_{1}$ is the modified Bessel function there exists a crack tip opening and the displacement on the crack plane outside the crack is not zero. It is possible to show that the integral and its first and second derivatives given by (31) are uniformly convergent which indicates that the crack closes smoothly in gradient elasticity. A detailed treatment of this problem has been published in [14].

\section{RESULTS AND DISCUSSION}

In this study definition of crack problems as a boundary value problem in the mathematical theory of elasticity is discussed. The discussion is carried out on mode III problem and in the light of this discussion the solutions of mode III problem within the framework of gradient-dependent and nonlocal elasticities are given.

First we claimed that the basic crack problems should be defined as a traction boundary value problem in elasticity and we outlined a method for solving the crack problems as traction boundary value problems which yield the same results those obtained by other methods. The method that we employed in this study will be reported in full detail elsewhere.

The solution of mode III crack within framework of nonlocal elasticity as a traction boundary value problem is summa- 
rized. According to the formalism that we have followed in this study we show that the exact solution of this problem can be expressed in terms of an infinite sum. A detailed treatment of this problem will also be reported elsewhere.

The solution of the same problem within the framework of gradient-dependent el asticity which has been published in. [14] displays some interesting features. The results that we obtained show that the crack faces do not close completely at its "physical tip" but they extend beyond it and close smoothly. This property indicates that the gradient elasticity is consistent with "Barenblatt's Theory" without requiring an extra condition. Moreover, since the second derivative of ( 30 ) is also uni formly convergent "dislocation emission" from crack tip is also included in gradient elasticity.

\section{REFERENCES}

1.G.C.Sih and H.Liebowitz, Mathematical Theories of Brittle Fracture, in FRACTURE vol.2, ed. H. Liebowitz, Academic Press, New York, 1968.

2. E. Kröner, 'Continuum Mechanics and Range of Atomic Cohesion Forces,'Proc. of the first Int. Conf. on Fracture, Sendai, Japan 1965.

3. I. A.Kunin, Elastic Media with Microstructure, Springer Verlag 1983.

4. D. Rogula, Nonlocal Theory of Material Media, Springer Verlag 1982 .

5. A. C. Eringen and D. G. B. Edelen, 'On Nonlocal Elasticity," Int. Journ. Engng. Soi. 10,1972.

6.C. Truesdel 1 and W. Noll, Encyclopedia of Physics, ed. S. Flügge, vol. II $/ 3$, Springer Verlag, 1965.

7.E. C. Aifantis, On the Microstructural Origin of Certain Inelastic Models,' Trans. ASME, J. Eng. Mat. Tech. $106,1984$.

8. H.M. Zbib and E.C. Aifantis, 'On the Structure and Width of Shear Bands', Scripta Metall. 22, 1988.

9. H. M. Zbib and E.C.Aifantis,'A Gradient-dependent Flow Theory of Plasticity: Application to Metal and Soil Instabilities'. Appl. Mech. Review, 42, 1989.

10. I. N. Sneddon, Fourier Transform, McGraw-Hid1, 1951.

11. I.N. Sneddon and M. Lowengrub, Crack Problems in the classial Theory of Elasticity, John wiley a Sons, Inc. New York. London-Sydney-Tor ont.o, 1969. 
12. G.N.Watson, Theory of Bessel Functions, The Cambridge University Press, Cambridge, 1952.

13. I.S. Gradshteyn and I.W. Ryzhik, Table of Integrals, Series and Products. Academic Press, New York-London, 1965.

14. B.S. Altan and E. C. Aifantis, Mode III Crack in Gradient Elasticity', Scripta Met. et Materialia, 1992 Caccepted for publication. 